\title{
BRCA2 Gene Mutation
}

National Cancer Institute

\section{Source}

National Cancer Institute. BRCA2 Gene Mutation. NCI Thesaurus. Code C19636.

A mutation that is typically a heritable, permanent change in the nucleotide sequence of the BRCA2 gene. Single nucleotide substitutions and small deletions or insertions (1-20 bases) account for the majority of mutations in the BRCA2 gene. Most of these alterations result in a truncated form of the breast cancer type 2 susceptibility protein. Mutations in the BRCA2 gene predispose males to breast cancer. 